INFECTION

\section{Renal IL-17 activity in candidiasis}

Disseminated Candida albicans infection is potentially fatal and frequently causes acute kidney injury. A recent study by Partha Biswas and colleagues provides new insights into the role of renal tubular epithelial cells (RTECs) in the defence against this fungal pathogen.

"We previously showed that IL-17 has a kidney-protective role during disseminated candidiasis," says Biswas. "However, there is controversy regarding the cellular source of IL-17 and the contribution of IL-17-responsive renal cells to immunity against $C$. albicans. Understanding these issues could lead to improvements in immune-based therapies for this poorly understood infection."

The researchers now report that TCR $\gamma \delta^{+}$ $T$ cells are the primary source of IL-17 in C. albicans-infected kidneys. Using chimeric mice, they demonstrate that IL-17 receptor $A$ (IL-17RA) expression on haematopoietic cells is not required for immunity to systemic C. albicans infection. However, infected mice with specific knockout of IL-17RA in RTECs (Il17 $\left.\mathrm{ra}^{\Delta R T E C}\right)$ showed more severe renal damage and reduced survival compared with wild-type controls. Their increased susceptibility to C. albicans infection was very similar to that of Il17ra-knockout mice, suggesting a key role of IL-17RA signalling in RTECs in the defence against this pathogen.

The researchers also show increased apoptosis and reduced activation of the kallikrein-kinin system (KKS) in RTECs in response to $C$. albicans infection in $1117 \mathrm{ra}^{\triangle \mathrm{RTEC}}$ mice compared with wild-type controls. Consistent with these findings, treatment with bradykinin - the end product of the KKS - significantly increased the survival of the infected Il17ra ${ }^{\triangle R T E C}$ mice.

"During systemic infection with C. albicans, IL-17 activity within the kidney is remarkably restricted to non-haematopoietic cells," concludes Biswas. "IL-17 is produced by $\mathrm{TCR} \gamma \delta^{+} \mathrm{T}$ cells and activates the KKS in RTECs, which is essential to limit apoptosis of renal cells and prevent kidney damage during fungal invasion. These findings may lead to the use of targeted therapies that impact the bradykinin pathway to counter renal damage during disseminated candidiasis."

Ellen F. Carney

ORIGINAL ARTICLE Ramani, K. et al. Unexpected kidneyrestricted role for $\mathrm{IL}-17$ receptor signaling in defense against systemic Candida albicans infection. JCl Insight https://doi. org/10.1172/jci.insight.98241 (2018)

\title{
GATM mutations cause mitochondrial abnormalities and kidney failure
}

A novel form of autosomal dominant tubuloglomerular disease is caused by mutations in the GATM gene that result in mitochondrial abnormalities, say Robert Kleta and colleagues. GATM encodes the mitochondrial enzyme glycine amidinotransferase (GATM), which has a role in creatine biosynthesis.

Using genome-wide linkage analysis and sequencing studies, the researchers identified four heterozygous missense mutations in GATM in 28 patients with Fanconi syndrome and kidney failure from five extended families. Kidney biopsy samples from these patients showed fibrosis and proximal tubule cells with extremely large mitochondria that contained pathological GATM aggregates. Overexpression of mutant GATM in a proximal tubule cell line resulted in a similar mitochondrial phenotype, whereas proximal tubule cells that expressed wild-type GATM had normal mitochondrial morphology.

To investigate the effect of the diseasecausing GATM mutations on the protein, the researchers performed in silico analyses.
They report that these mutations result in the formation of an interaction site that could enable GATM to form linear multimers consistent with the mitochondrial aggregates.

Finally, the researchers show that overexpression of mutant GATM in proximal tubule cells impaired mitochondrial turnover and led to increased production of reactive oxygen species, initiation of an inflammatory response, release of profibrotic factors and increased cell death.

"We speculate that this renal proximal tubular mitochondrial pathology initiates a response from the inflammasome, with subsequent development of kidney fibrosis," conclude the researchers. "The disease also illustrates the critical role that mitochondria can play in initiating devastating profibrotic signalling cascades."

Ellen F. Carney

ORIGINAL ARTICLE Reichold, M. et al. Glycine

amidinotransferase (GATM), renal Fanconi syndrome and kidney failure. J. Am. Soc. Nephrol. https://doi.org/10.1681/ ASN.2017111179 (2018)

\section{GENETICS}

\section{GWAS highlights challenges associated with identification of DKD risk variants}

Diabetic kidney disease (DKD) is a serious complication of diabetes. Substantial between-patient variation exists in the progression and severity of DKD; however, the factors that contribute to this variability are unknown. Several lines of evidence suggest that underlying genetic components influence the risk of DKD, but few loci identified in genome-wide association studies (GWAS) have been confirmed. New findings from the largest GWAS to date of DKD in patients with type 2 diabetes mellitus (T2DM) highlight the challenges of identifying risk variants associated with DKD. "Sample size is key, and previous results from GWAS of DKD in T2DM and T1DM have been underpowered, with reported signals showing limited replication," says Mark McCarthy. "We set out to perform a substantially larger study than previous studies, but despite samples sizes that exceeded 40,000 , the yield of novel discoveries was modest."

Given uncertainties over which of the various DKD stages might be the most tractable for genetic dissection, the researchers separated cases into eight phenotypic classes. Using this approach, they identified a novel locus, GABRR1 (centred on rs9942471), associated with microalbuminuria in European individuals with T2DM, which was not present in individuals of Asian ancestry, but they caution the need for replication studies to confirm this association. They could not confirm previously reported genetic associations, except for UMOD and PRKAG, which were associated with estimated glomerular filtration rate. "We did, however, find some evidence that genetic variants influencing insulin action and obesity are also associated with DKD risk, reinforcing evidence of a connection between DKD and these traits from other sources," says McCarthy.

Susan J. Allison

ORIGINAL ARTICLE van Zuydam, N. R. et al. A genome-wide association study of diabetic kidney disease in subjects with type 2 diabetes. Diabetes https://doi.org/10.2337/db17-0914 (2018) 